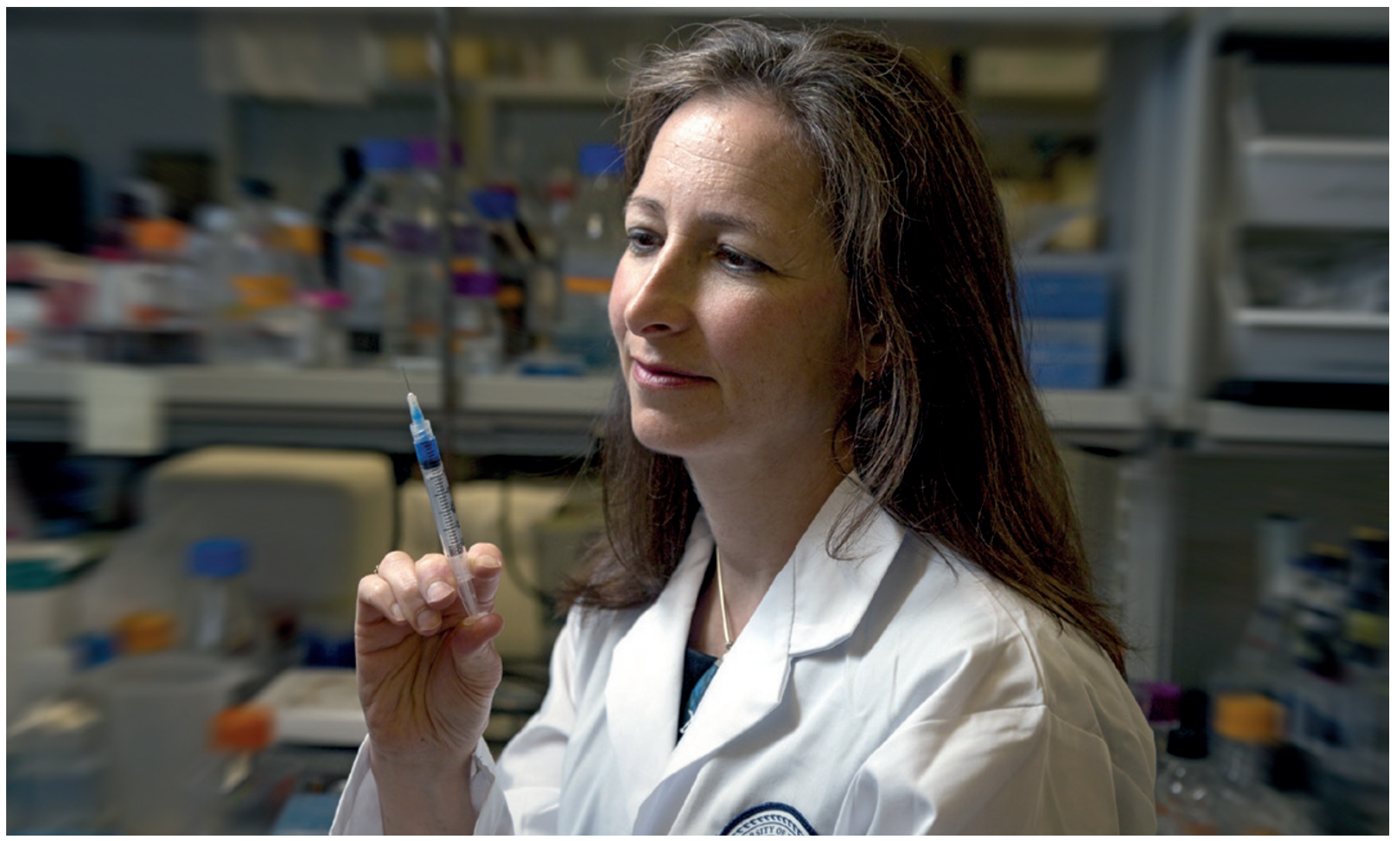

Molly Shoichet has developed a polymer than can deliver drugs to the brain with the minimum of damage.

DRUG DELIVERY

\title{
Brain food
}

\section{The key to stroke recovery is to coax the brain cells to heal without creating more damage in the process. A clever delivery system may just do the trick.}

\section{BY HANNAH HOAG}

$\mathrm{M}$ olly Shoichet searches her desk for a sandwich-sized zip-up bag. Stowed in the bag is a syringe containing roughly 2 millilitres of a rich-turquoise fluid. She pops the cap off the needle and pushes the plunger gently until a small bead of the material emerges. She tilts the syringe upside down, but the tiny bulb stays put. Almost instantly, the liquid had transformed into a gel - one that Shoichet hopes will be able to deliver drugs directly to the brain.

The material in the syringe is a three-dimensional network of polymers called a hydrogel. It is a viscous fluid when flowing down a narrowgauged needle, but becomes a gel once it has been injected into the body. "It's important that it gels quickly, so that it doesn't disperse too widely," says Shoichet, a polymer chemist and biomedical engineer at the University of Toronto in Canada.

Shoichet designed the hydrogel to aid the repair of brain tissue after a stroke deprives the brain of oxygen. The brain has self-renewing cells - called neural stem cells - that can differentiate into multiple cell types ${ }^{1}$. After a stroke, these cells move towards the damage to repair it ${ }^{2}$, but as a general rule, not enough cells mature into functioning tissue ${ }^{3}$, so lasting signs of damage remain. To coax the brain to heal, researchers face two main challenges: how to keep the cells alive long enough for them to mature and how to integrate them into the neural circuitry so that they can repair the tissue and restore function.

The hydrogel strategy aims to solve both problems. Suspended within the gel are two cellular growth factors, both neatly encapsulated in polymer shells that control the timing of their release. One factor causes the stem cells to multiply and the other prompts them to differentiate into the neurons and glial cells that will

\section{ONATURE.COM}

For more on the potential of hydrogels, visit:

go.nature.com/pkyrj1 become the new brain tissue. Like a gardener who prepares for planting by mixing the best soil and adding the right amount of fertilizer, Shoichet is creating flexible dressings that feed and cultivate immature brain cells.

Shoichet began working on stroke in 2008 . Her lab had been collaborating with Charles Tator, a neurosurgeon at the University of Toronto, and Dimpy Gupta, his graduate student, to design a hydrogel that could be used to treat spinal-cord injury ${ }^{4}$. The material had to be liquid enough to flow through an ultrafine needle, but solid enough to stay in place after being injected into the space between the spinal cord and the thick membrane that surrounds it. The substance she developed contains hyaluronan to keep it fluid inside the syringe, and methylcellulose to allow it to gel once it emerges. Cindi Morshead, a stem-cell biologist at the University of Toronto who was working on another part of the spinal-cord injury project, suggested that they try a similar approach for the stroke-injured brain. 
In 2006, Morshead had used infusions of two growth factors to coax the brains of stroke-injured rats to heal ${ }^{5}$. After letting the stroke take hold for 4 days, she used a hollow tube called a cannula to infuse the brain with epidermal growth factor for 7 days and prompt the small number of stem cells in the brain to proliferate and migrate to the site of the injury. She then infused erythropoietin for 7 days to encourage the cells to differentiate into neurons (see 'Right on target'). The combination worked, increasing the number of neurons at the site of the injury and reducing the size of the injured area.

\section{AVOID HEALTHY TISSUE}

The trouble was that the technique required surgery that damaged healthy brain tissue. "It was too invasive," says Morshead. She wondered whether Shoichet's hydrogel would eliminate the need for the cannula.

Shoichet's first challenge was to find a way to allow for both sequential and sustained release of the growth factors: one needed to be released 4 days after the stroke, the second 7 days later, and each had to be released over a week.

One way to control the delivery is to coat the drugs with a polymer that takes time to break down in the body. This was one of Shoichet's areas of expertise. She had earned her undergraduate degree at the Massachusetts Institute of Technology in Cambridge, where she says she "got really excited about polymers and their use in medicine and pharmaceutical chemistry".

After completing her PhD in polymer science and engineering at the University of Massachusetts Amherst, she joined a start-up called Cytotherapeutics in Providence, Rhode Island, where she designed polymers to encapsulate cells for the treatment of Parkinson's disease, diabetes and chronic pain. It was there that her materials expertise fell under the influence of life science. "I was surrounded by really smart biologists," she says. "Rather than take something off the shelf and see if it works, I could design a material to produce the response they wanted."

For the hydrogel, Shoichet began by wrapping nanometre-sized droplets of each of the two growth factors in polymers. She then added a layer of polysebacic acid to the erythropoietin bundle so that the drug would not be released at the same time as the endothelial growth factor. Then she suspended the beads in the hydrogel ${ }^{6}$. With the challenge of developing a gel behind them, she moved on to test it in a mouse model of stroke.

Shoichet, Morshead and their collaborators fixed a small disk with an opening over a hole in the skull and dripped the hydrogel directly onto the brains of the stroke-injured mice. It worked: the brains had smaller areas of dead tissue, more living neurons in the damaged area and less inflammation compared with mice that received the growth factors through the cannula ${ }^{6}$. And because the 4-day

\section{RIGHT ON TARGET}

A hydrogel polymer may allow targeted, time-sensitive delivery of brain-repairing drugs.

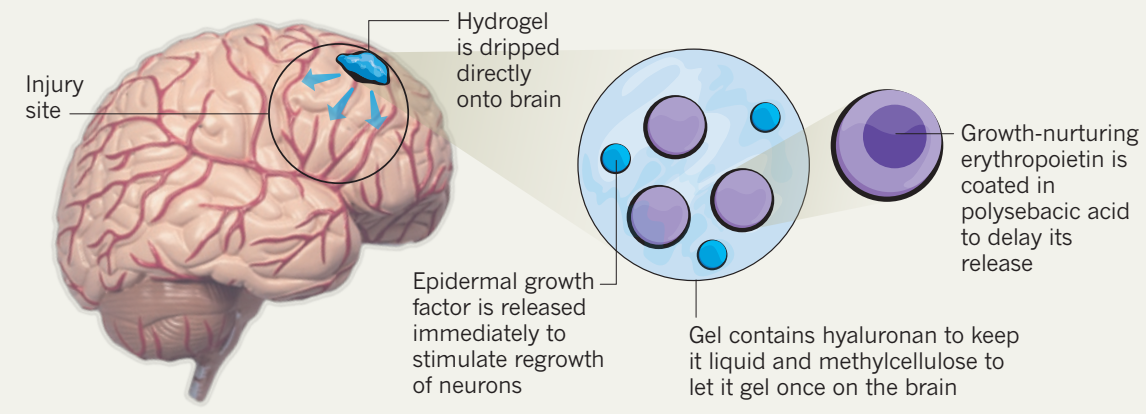

\begin{tabular}{|l|l|l|l|l|}
\hline \multicolumn{2}{|c|}{ Release profile } & \multicolumn{2}{l}{ Epidermal growth factor } & Erythropoietin \\
\hline Stroke & Day 4 & Day 11 & Day 18
\end{tabular}

delay meant that very few cells were still alive, the living neurons the researchers saw in the brains were likely to be new cells rather than repaired ones.

Although Shoichet and Morshead are most interested in using the brain's existing stem cells to repair itself after stroke, they have also experimented with stem-cell transplantation. In unpublished work, they have mixed the hydrogel with neural stem cells from a healthy adult mouse, then injected these directly into the brain of a stroke-injured mouse. The cells lived longer when introduced through the hydrogel than when transplanted on their own, but the results still fall short of Shoichet's aims. “There aren't as many neurons as we'd like," she says. She hopes that the number of neurons can be boosted by allowing the stem cells to differentiate before transplanting them.

\section{YOUNG AT HEART}

Shoichet and Morshead remain optimistic about their hydrogel approach. It avoids the complications faced by intravenous administration of stem cells or drugs, an approach that is being tested in early clinical trials. The main problem with intravenous administration is that the blood-brain barrier limits the passage of growth factors into the brain, so higher drug doses are often needed, leading to unwanted side effects. It also avoids the damage to healthy tissue caused by infusing the drugs through a cannula.

Shoichet and Morshead are now tweaking the hydrogel's components to improve its performance. They have added ciclosporin, a drug that promotes the survival of neural precursor cells $^{7}$. They are also looking for other molecules and strategies that can help to repair the damaged site, such as breaking down the scar of glial tissue that typically forms after a stroke.

Another goal is to see whether the hydrogel therapy works in older mammals because "that is the most prominent population that will suffer from these deficits, and the older body doesn't work as well as the younger one", says Morshead.

Other researchers are also developing biomaterials to repair the stroke-injured brain. The combination of substances such as polymers, growth factors and cytokines (small proteins that act as cell signals) varies among the research groups, as does the method of application. None replicates Shoichet and Morshead's less-invasive hydrogel approach, but some are following a similar path. For example, a group at the Nagoya City University Graduate School of Medical Sciences in Japan has injected the brains of mice with gelatin microspheres laced with a growth factor. Insulin growth factor 1 increased the number of neurons in a part of the brain known as the subventricular zone; and hepatocyte growth factor increased the number of neurons migrating from this region to the damaged site ${ }^{8}$.

For scientists focused on regenerating nerve tissue, getting beyond the brain's protective barriers can be a challenge. But Shoichet and Morshead think that they are getting close to finding a way to help the brain heal itself. "Celltransplant strategies are costly and complex," says Shoichet. "It would be great if we could take advantage of what was already there."

Hannah Hoag is a freelance science writer in Toronto, Canada.

1. Reynolds, B. A. \& Weiss, S. Science 255, 1707-1710 (1992)

2. Jin, K. et al. Proc. Natl Acad. Sci. USA 103, 13198-13202 (2006)

3. Abrous, D. N., Koehl, M. \& Le Moal, M. Physiol. Rev. 85, 523-569 (2005).

4. Gupta, D., Tator, C. H. \& Shoichet, M. S. Biomaterials 27, 2370-2379 (2006).

5. Kolb, B. et al. J. Cereb. Blood Flow Metab. 27, 983-997 (2007).

6. Wang, Y., Cooke, M. J., Sachewsky, N., Morshead, C. M. \& Shoichet, M. S. J. Control. Release 172, 1-11 (2013).

7. Caicco, M. J. et al. J. Control. Release 166, 197-202 (2013).

8. Nakaguchi, K. et al. Stem Cells Int. http://dx.doi. org/10.1155/2012/915160 (2012). 